\title{
The Melting Temperature of Thermally Reversible Gel. I. Poly(vinyl chloride)-Organic Solvent Gels
}

\author{
Akira Takahashi, Takeshi Nakamura, and Ikumi Kagawa \\ Department of Applied Chemistry, Nagoya University, \\ Nagoya, Japan.
}

(Received July 13, 1971)

\begin{abstract}
The melting temperatures of gels that were formed from dilute solutions of poly(vinyl chloride) in anisole, dioxane, and ethylene dichloride were measured over a volume fraction range from 0.02 to 0.05 . Five fractions of poly(vinyl chloride) with molecular wəights ranging from 67000 and 200000 having almost identical syndiotacticity $\alpha=0.5$, were used. The linear relationship was found for each solvent between the reciprocal absolute temperature of melting and the logarithm of $v_{2} x$, where $v_{2}$ is the volume fraction of the polymer and $x$ is its degree of polymerization. The X-ray diffraction pattern revealed that the gel was crystalline, thus confirming the supposition that the "cross links" are crystallites. The results were compared with the theory of melting point depression for copolymer-diluent systems. However, the theory did not fit the experimental results and it was concluded that the melting point of gel does not correspond to the thermodynamic singurality. An expression was derived from the condition of gel formation and the free energy of crystallite formation, with the supposition that the "cross links" are crystallites. The expression was compared with the experimental results, and it was found that it fairly well represents the experimentally observed gel melting points as a function of molecular weight, polymer concentration and the thermodynamic nature of the solvent. The syndiotactic sequence length in the crystallite was estimated to be $10-12$.
\end{abstract}

KEY WORDS Gel / Melting Temperature of Gel / Poly(vinyl chloride) / Thermally Reversible Gel /

Several years ago, we observed that dilue solutions of poly(vinyl chloride) in ethyl acetoacetate are turned into gel on cooling and the gelation is reversible on warming. ${ }^{1}$ However, the gelation phenomena is not confined the above polymer-solvent pair. Walter ${ }^{2}$ studied the poly(vinyl chloride)-dioctyl-phthalate gel. The systems of poly(vinyl chloride)-anisole, dioxane ${ }^{3}$ and ethylene dichloride which we wish to report herein also exhibit thermally reversible gelation. Very recently, Harrison, Morgan, and Park $^{3}$ have reported the melting temperature of poly(vinyl chloride)-dioxan gel. Reversible gelation is by no means a unique property of poly(vinyl chloride), for this behavior has been observed for several polymer-diluent systems. ${ }^{4}$ The well-known examples are the systems of gelatin-water and agar-water.

It is well known ${ }^{5}$ from simple geometrical considerations that at least two cross-links per poly- mer chain are needed to form a three dimensional network. Taking into account this requirement for gel formation, and by treating the cross-link formation as a chemical equilibrium, Eldridge and Ferry ${ }^{6}$ derived a useful expression concerning the melting point of gel $T_{\mathrm{m}} \mathrm{g}$ and polymer concentration $C$

$$
\ln C=\frac{\Delta H_{\mathrm{m}}}{R T_{\mathrm{m}^{\mathrm{g}}}^{\mathrm{g}}}+\text { (const) }
$$

Here $C$ is expressed in $\mathrm{g} / l$ and $\Delta H_{\mathrm{m}}$ is the energy associated with the dissociation of a network in gel.

Although many papers on gelation phenomena have appeared in the past two decades, the thermodynamics and kinetics of this sol-gel transition are not completely explained. In the investigations reported in the past, most of the attention has been focused on the relationship between the melting point of gel and the polymer con- 
centration, and only a few experimental studies have been made on the relationship between $T_{\mathrm{m}} \mathrm{g}$ and molecular weights of samples. The effect of solvent properties on $T_{\mathrm{m}} \mathrm{g}$ has seldom been studied.

It is the purpose of this paper to clarify the relationship existing among the gel melting points, molecular weights, polymer concentrations, and solvent properties by using poly(vinyl chloride) gels that are formed in anisole, dioxane, and ethylene dichloride.

\section{EXPERIMENTAL}

\section{Materials}

Five samples of poly(vinyl chloride)(PVC), which differ in molecular weights but with almost the same syndiotacticity, have been used. All the polymers were purified by solution in tetrahydrofuran and precipitation in methanol, and dried in vacuo for three days. Anisole, dioxane, and ethylene dichloride (EDC) were used as solvents for gelation, and were purified by distillation before use.

\section{Characterization of Polymers}

Molecular weights of the PVC samples were determined by viscosity measurement in tetrahydrofuran at $25^{\circ} \mathrm{C}$ using the viscosity equation ${ }^{1}$

$$
[\eta]=3.98 \times 10^{-4} M_{w}{ }^{0.69}
$$

Infrared spectroscopy was employed to measure the syndiotacticity of each polymer. Following the method of Germar, Hellwege, and Johnson, ${ }^{7}$ each polymer was dissolved in $1,1,2,2-$ tetrachloroethane as $25 \mathrm{~g} / l$ solution. Using a cell $0.5-\mathrm{mm}$ thick, the optical densities $D$ at $1434 \mathrm{~cm}^{-1}$ and $1428 \mathrm{~cm}^{-1}$ of the solution were measured at two temperatures $298^{\circ} \mathrm{K}$ and $350^{\circ} \mathrm{K}$ by a JASCO Infrared Spectrometer Model G. The ratios defined by $\lambda=D_{1434} / D_{1428}$ at two temperatures, $T_{1}$ and $T_{2}$, are related to the energy difference $\Delta E$ between two syndiotactic conformations by the following equation.
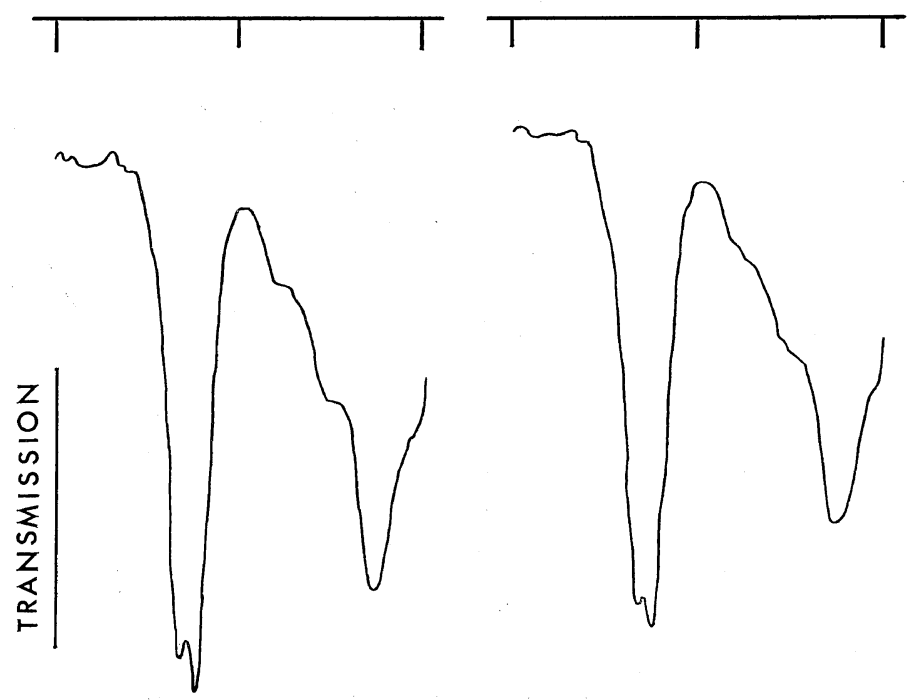

$25^{\circ} \mathrm{C}$

$77^{\circ} \mathrm{C}$

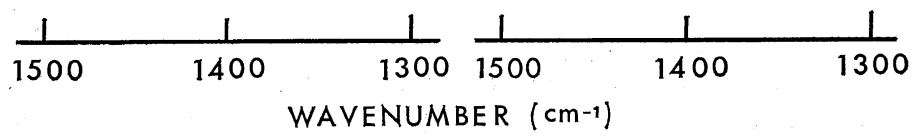

Figure 1. Infrared spectra for $\mathrm{CH}_{2}$-scissor vibration in poly(vinyl chloride) sample No. 5 at 25 and $77^{\circ} \mathrm{C}$. 


$$
\frac{1+\lambda_{1}}{1+\lambda_{2}}=\frac{1+\exp \left(-\Delta E / R T_{1}\right)}{1+\exp \left(-\Delta E / R T_{2}\right)}
$$

An example of the IR measurement is shown in Figure 1, and the quantity $\left(1+\lambda_{1}\right) /\left(1+\lambda_{2}\right)$ was calculated from the observed values of $\lambda$ and then the energy difference $\Delta E$ was obtained from eq 3. The syndiotacticity $\alpha$ of the samples was calculated by the relationship

$$
\alpha=\frac{1+\exp (-\Delta E / R T)}{1+\lambda}
$$

The details of five samples are given in Table I.

Table I. Molecular weight and syndiotacticity of poly(vinyl chloride)

\begin{tabular}{ccccc}
\hline $\begin{array}{c}\text { Sample } \\
\text { no. }\end{array}$ & $M \times 10^{-4}$ & $D P$ & $\begin{array}{c}\Delta E, \\
\mathrm{cal} / \mathrm{mol}\end{array}$ & $\alpha$ \\
\hline 1 & 6.72 & 1080 & 3580 & 0.507 \\
2 & 9.57 & 1530 & 3150 & 0.52 \\
3 & 10.36 & 1660 & 2160 & $0.53_{3}$ \\
4 & 13.1 & 2100 & 4860 & $0.51_{5}$ \\
5 & 20.6 & 3290 & 2230 & $0.53_{4}$ \\
\hline
\end{tabular}

\section{Melting Temperature of Gel}

Each gel was made by stirring a mixture of polymer and dry solvent in a flask at a temperature above the gel melting point. The gel melting point $T_{\mathrm{m}} \mathrm{g}$ was determined in the viscometer-like apparatus shown in Figure $2 .^{2}$ This was filled with molten gel, and the gel was allowed to set with negative pressure on one limb of the apparatus so that the height $\Delta h$ between the two menisci in the limbs was $4-6 \mathrm{~cm}$. Gelation of the molten PVC-anisole and PVC-EDC gels was carried out at $0^{\circ} \mathrm{C}$ for $22 \mathrm{hr}$ while gelation of the PVC-dioxane gels was conducted at $15^{\circ} \mathrm{C}$ for $20 \mathrm{hr}$. Then the temperature was increased at a rate of $10^{\circ} \mathrm{C} / \mathrm{hr}$, until flow occurred in the limbs as was observed by a fairly rapid decrease in $\Delta h$. If the sol produced on the melting of the gel were a Newtonian fluid of constant viscosity, $\Delta h$ should decrease exponentially with time. The intersection of the two linear portions of the logarithmic plots shown in Figure 3 affords an accurate measurement of the gel melting point. The change of the initial values of $\Delta h$ from 2 to $8 \mathrm{~cm}$ has no effect on the observed $T_{\mathrm{m}} \mathrm{g}$ within experimental error when the gels were formed at the same time

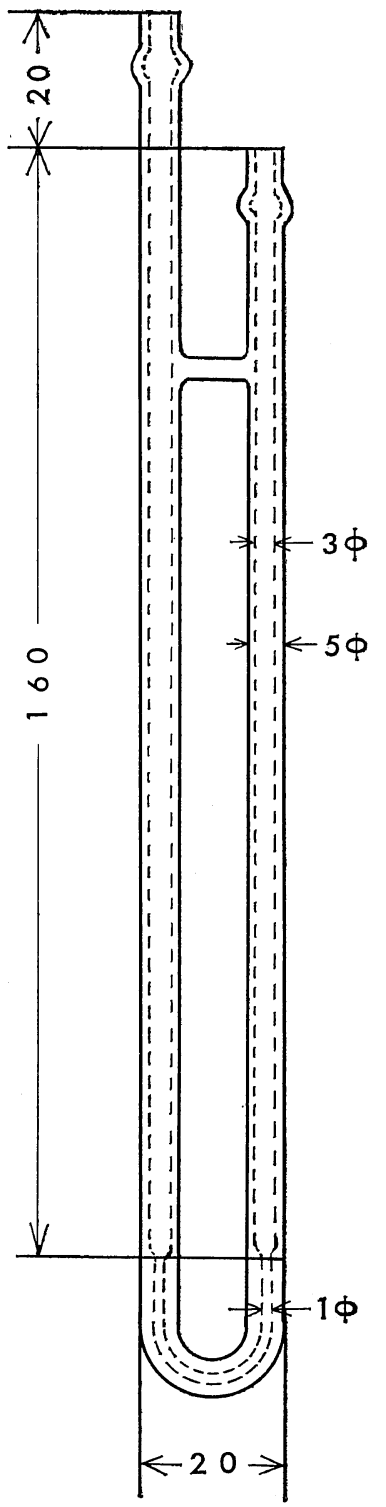

Figure 2. Gel melting temperature apparatus.

from the same molten gel. However, after recording the melting point of the first gel, the second gel was formed again under the same experimental condition. The melting point of the second gel was measured again, and the reproducibility of the melting point was within $1^{\circ} \mathrm{C}$.

\section{$X$-ray Diffraction of Gel}

A molten gel was injected into a silica fine 


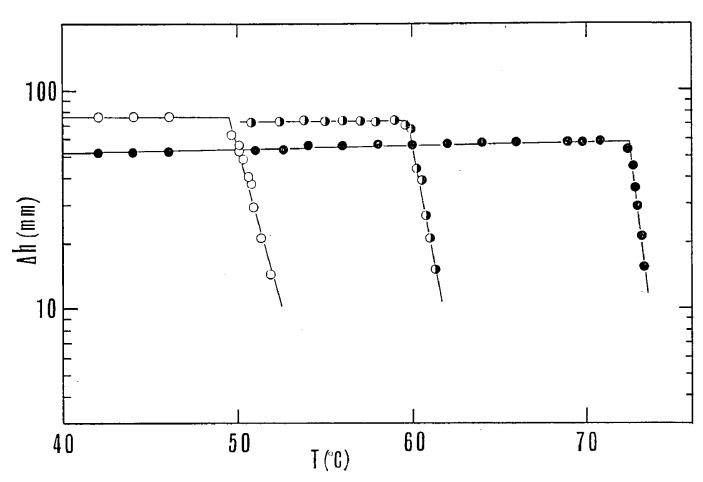

Figure 3. Examples for determination of $T_{\mathrm{m}} \mathrm{g}$ : sample No. 2 in dioxane; $\bigcirc, v_{2} 0.0218 ; v_{2} 0.028$; ○, $v_{2} 0.0354$.

capillary (1-mm inner diameter) by a hypodemic needle and gelation was conducted at $0^{\circ} \mathrm{C}$ for $20 \mathrm{hr}$ in the capillary. The capillary was mounted on a Toshiba X-ray diffraction camera and the X-ray diffraction pattern was obtained using $\mathrm{CuK}_{\alpha}$ point beam.

\section{RESULTS}

\section{$X$-ray Diffraction}

A typical $X$-ray diffraction pattern of PVC (No. 1)-EDC gel is shown in Figure 4. The

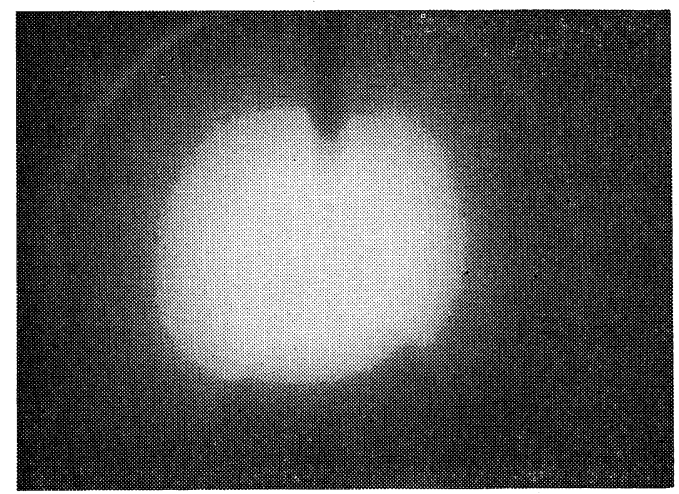

Figure 4, X-ray diffraction pattern of PVC-dioxane gel: sample No. $1 ; C=4 \mathrm{~g} / 100 \mathrm{~m} l$.

polymer concentration in the gel was $4 \mathrm{~g} / 100 \mathrm{ml}$. Two diffraction rings which correspond $2 \theta=44^{\circ}$ and $2 \theta=55^{\circ}$ or $d=1.66 \AA$ and $d=2.06 \AA$ are observed, though their intensity was very weak. It is apparent that the gel is crystalline. Moreover, the gel was almost transparent and no precipitate existed in the gel. For our purpose, it was sufficient to prove that the gel is crystalline. Therefore, we have not gone further into detail concerning the problem of the structure of the crystalline regions.

\section{Polymer Concentration and $T_{\mathrm{m}} \mathrm{g}$}

The values of $T_{\mathrm{nt}}{ }^{\mathrm{g}}$ of gels for specified solvent increase with the concentration of the polymer at constant molecular weight. Ferry and Eldridge $^{6}$ have treated gel formation as a chemical equilibrim. As was summarized by Harrison, Morgan, and Park, ${ }^{3}$ their assumption is (a) that the concentration of free network linking loci is proportional to the polymer concentration, (b) that the concentration of cross-links is proportional to the concentration of polymer molecules at the gel point and (c) that the binary association equilibrium between cross-links and the free network linking loci can be related to the temperature using the van't Hoff isochore. Thus, they obtained eq 1 . An example of plot of eq 1 is shown in Figure 5, in which $1 / T_{\mathrm{m}} \mathrm{g}$ is plotted against the logarithm of the volume fraction of polymer $v_{2}$ in the gel. It is apparent that the experimental results are in reasonable accord with eq 1. As another example, the same plots

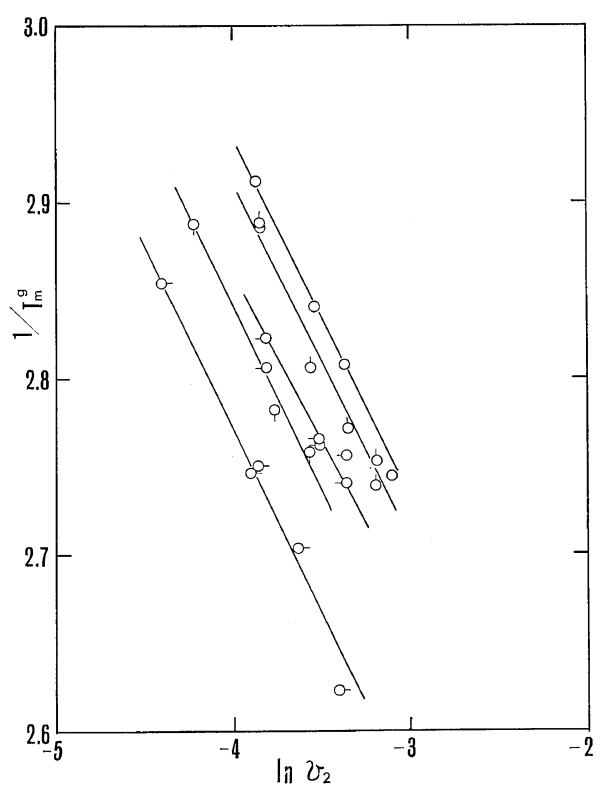

Figure 5. Plots of $1 / T_{\mathrm{m}^{\mathrm{g}}}$ vs. $\ln v_{2}$. System, PVCanisole. Molecular weights: $\bigcirc, 6.72 \times 10^{-4}$; $\mathrm{O}$, $9.59 \times 10^{-4} ;-\bigcirc, 10.36 \times 10^{-4}$; ○, $13.1 \times 10^{-4}$; and $\bigcirc-$, $20.6 \times 10^{-4}$. 


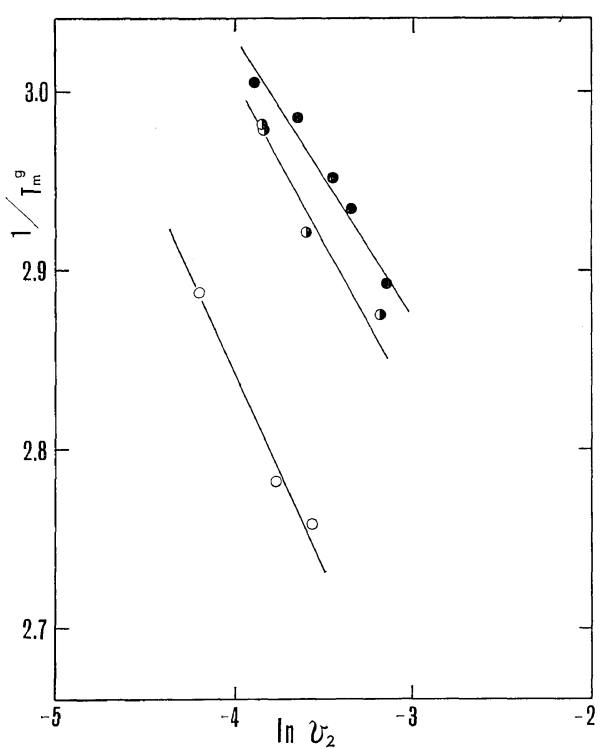

Figure 6. Plots of $1 / T_{\mathrm{m}} \mathrm{g}$ vs. $\ln v_{2}$ for three PVC gels: molecular weight of the sample, 131000; $\bigcirc$, PVC-anisole; (1, PVC-dioxane; O, PVC-EDC.

are shown in Figure 6, for which the different solvents are used, but the same molecular weight sample is employed.

Molecular Weight, Solvent, Stereoregularity, and $T_{\mathrm{m}}^{\mathrm{g}}$

Eldridge and Ferry ${ }^{5}$ have also derived the relationship between $T_{\mathrm{m}}^{\mathrm{g}}$ and weight average molecular weight $M_{w}$ of the polymer as

$$
\ln M_{w}=\frac{\Delta H_{\mathrm{m}}}{7 R T_{\mathrm{m}} \mathrm{g}}+(\text { const })_{2}
$$

Combining eq 5 with eq 1 , we obtain

$$
\ln C M_{w}=\frac{8}{7} \cdot \frac{\Delta H_{\mathrm{m}}}{R T_{\mathrm{m}} \mathrm{g}}+(\text { const })_{3}
$$

If $\Delta H_{\mathrm{m}}$ were independent of both the molecular weight and the polymer concentration of the gels, the plot of $1 / T_{\mathrm{m}}^{\mathrm{g}} v s . \ln C M_{w}$ (or $\ln v_{2} x$, where $v_{2}$ is the volume fraction of polymer and $x$ is its degree of polymerization) should fall on a straight line for a specified solvent. Figure 7 shows that the plots of $1 / T_{\mathrm{m}} \mathrm{g} v s$. on $\ln v_{2} x$ for the three gelling systems are in reasonable agreement with this relationship, though the scattering of the experimental points is fairly appreciable. However, considering the fact that the scattering of the experimental points is within an experimental reproducibility of $T_{\mathrm{m}} \mathrm{g}$ of each gel, we may consider that the relationship of eq 6 is satisfied in the present case.

The intercepts of the plots, i.e., the values of (const) ${ }_{3}$ are shown in Table II. Their order is dioxane $\simeq \mathrm{EDC}>$ anisole. Doty and $\mathrm{Zable}^{8}$ have determined the interaction parameter $\chi_{1}$ for many pairs of PVC and organic solvents. The $\chi_{1}$ parameters determined for three solvent systems at $76^{\circ} \mathrm{C}$ are as follows: PVC-EDC, 0.43; PVC -dioxane, 0.455; and PVC-anisole, 0.49. Thus it appears that there is a correlation between the value of (const $)_{3}$ and the value of $\chi_{1}$ parameter.

Since our samples have almost the same syndiotacticity, no conclusion concerning the effect of the polymer regurality upon the melting temperature of gel has been reached.

\section{DISCUSSION}

The X-ray diffraction pattern indicates that the PVC gels are crystalline. Moreover, no precipirate was observed in the gel, and the gels were almost transparent to the naked-eye, but turbid when they were examined in a light scattering photometer. Therefore, we may consider that the gels consist of microcrystalline regions which pervade the whole volume. This conclusion has already been reached by light scattering studies on gelatin gels by Boedtker and Doty $^{9}$ and also on cellulose nitrate gels by Newman, Krigbaum, and Carpenter. ${ }^{10}$ Since the gel

Table II. The estimation of $\zeta$ and $\sigma_{e c}$

\begin{tabular}{lccccc}
\hline \multirow{2}{*}{ System } & \multicolumn{2}{c}{ Obsd } & & \\
\cline { 2 - 5 } & $\begin{array}{c}\text { Intercept, } \\
{ }^{\circ} \mathrm{K}^{-1} \times 10^{3}\end{array}$ & $\begin{array}{c}\text { Slope, } \\
\text { cal } / \mathrm{mol} \times 10^{-4}\end{array}$ & $\left.\zeta B^{\prime} V_{A}\right)$, & $\sigma_{e c}$, cal $/ \mathrm{mol}$ \\
\hline PVC-Anisole & $3.4_{7} \times 10^{3}$ & $1.7_{7}$ & $1.1_{2}$ & $11.7^{\prime}$ & -437 \\
PVC-Dioxane & $3.8_{3}$ & 2.3 & $0.8_{6}$ & 9.4 & -150 \\
PVC-EDC & $3.8_{6}$ & 2.2 & 0.89 & 9.6 & +66 \\
\hline
\end{tabular}




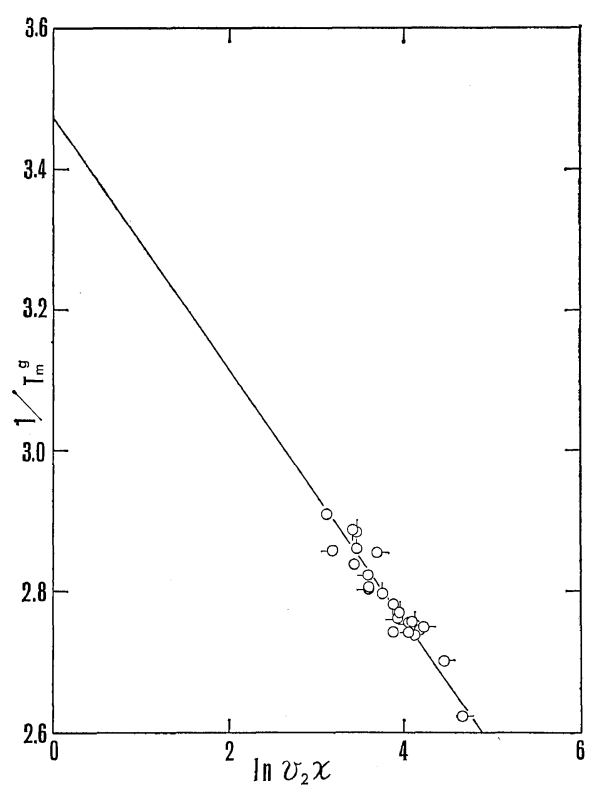

(a)

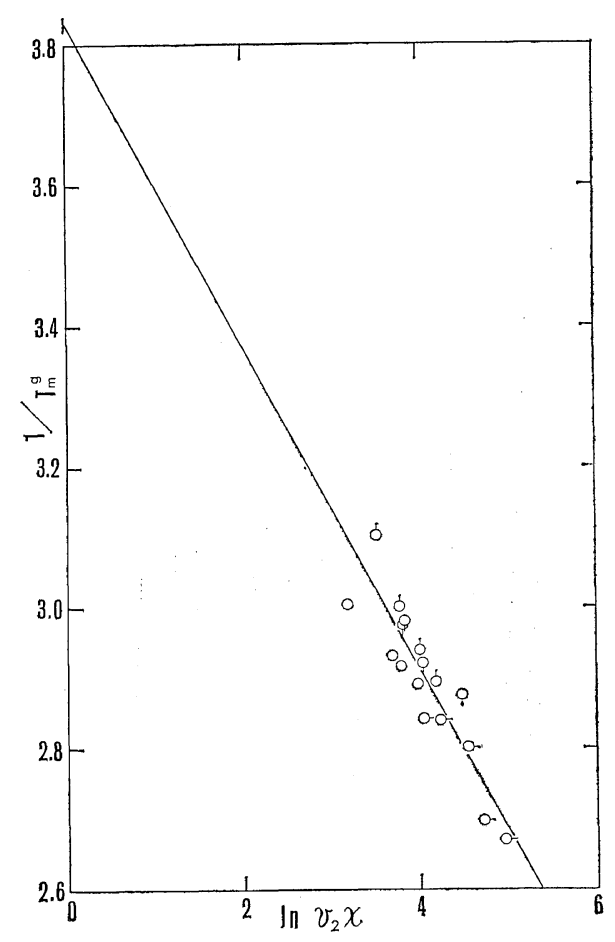

(b)

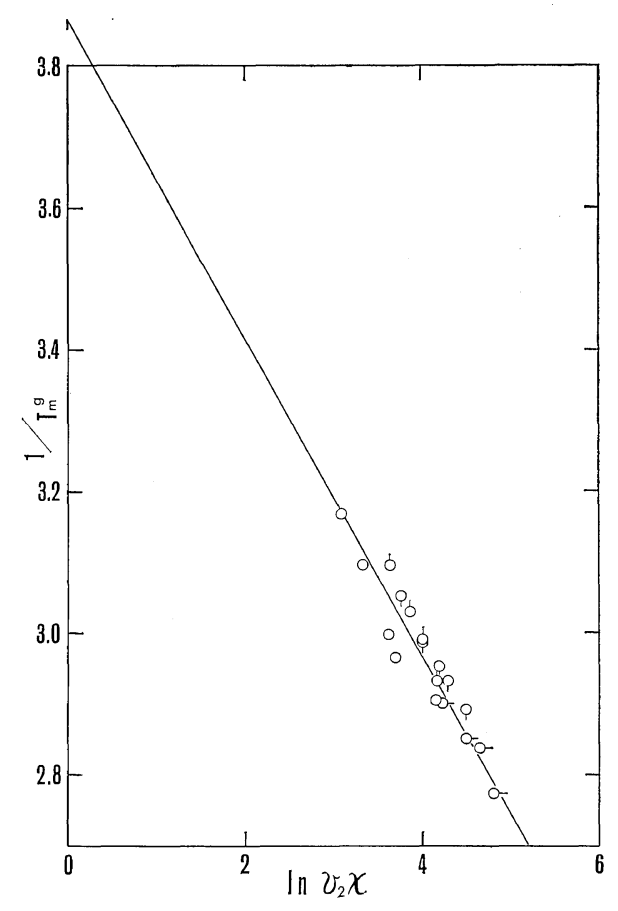

(c)

Figure 7. Plots of $1 / T_{\mathrm{m}} \mathrm{g}$ vs. $\ln v_{2} x$ for three PVC gels: (a) PVC-anisole; (b) PVC-dioxane; (c) PVC-EDC. The symbols for molecular weight are the same as in Figure 5.

point is the point at which the infinite three dimensional network first appears in the system through interconnection of primary molecules by cross-linking points, it is considered that the microcrystalline regions which pervade in the system serve as the cross-linking points. However, distinction must be made between the gel formed by crystallization and the gels formed by chemical reactions without phase change.

It has been pointed out ${ }^{11}$ that two important factors are conductive for gel formation. One is that the copolymeric character, which adjusts both the longitudinal and lateral growth of the microcrystalline regions, leads to the gel formation instead of the appearance of separate crystalline precipitates from the solution. The other is the magnitude and sign of the free energy of dilution relative to that of fusion. These factors also govern the growth of the crystalline region in dilute solution.

In order to get further insight into the gela- 
tion problem, we cnnsidered as a starting point the melting point depression of a copolymerdiluent system as formulated by Flory. ${ }^{12}$ The free energy of fusion of a crystallite, which contains $\zeta$ repeating crystallizable units $A$ in length, can be expressed as

$$
\begin{aligned}
& \frac{\Delta F_{\mathrm{f}}}{x N}=(1-\omega) \Delta f_{\mathrm{u}}+R T\left\{\left[\left(\frac{\bar{V}}{V_{1}}\right)\left(\frac{1-v_{2}}{v_{2}}\right)\right.\right. \\
& \left.+\frac{1}{x}\right] \ln \left[1-v_{2}\left(\frac{V_{\mathrm{A}}}{\bar{V}}\right)(1-\omega)\right] \\
& \quad+\frac{(1-\omega)}{\zeta}\left[\ln v_{2}-\frac{2 \sigma_{\mathrm{ec}}}{R T}+\ln \left(\frac{x-\zeta+1}{x}\right)\right] \\
& \left.\quad+(1-\omega) \ln X_{\mathrm{A}}+\chi^{\prime} \frac{\left[1-v_{2} X_{\mathrm{A}}\left(V_{\mathrm{A}} / \bar{V}\right)\right](1-\omega)}{\left[1-v_{2}\left(V_{\mathrm{A}} / \bar{V}\right)(1-\omega)\right]}\right\}
\end{aligned}
$$

Here $\Delta f_{\mathrm{u}}$ is the free energy of fustion per repeating unit in a pure crystal, $x$ is the number of repeating units per polymer chain, $N$ is the number of polymer molecules in the system and $v_{2}$ is the volume fraction of polymer, $\omega$ is the fraction of the polymer that is noncrystalline, $X_{\mathrm{A}}$ is the mol fraction of crystallizable unit, $\chi^{\prime}$ is the thermodynamic interaction parameter defined by $\chi^{\prime}=B^{\prime} V_{\mathrm{A}} / R T, \sigma_{\mathrm{ec}}$ is the end interfacial free energy per crystalline sequence and $V_{\mathrm{A}}, \bar{V}$, and $V_{1}$ are the molar volumes of crystallizable unit, of average copolymer unit and of solvent, respectively.

The melting temperature of a nonequilibrium crystallite of size $\zeta$ is obtained by setting $\partial \Delta F_{\mathrm{f}} /$ $\partial \omega)_{\zeta}=0$, and then by letting $\omega \rightarrow 1$ in the resulting expression. It is found that

$$
\begin{aligned}
& \frac{1}{T_{\mathrm{m}}}-\frac{1}{T_{\mathrm{m}}{ }^{0}}=\frac{R}{\Delta h_{\mathrm{u}}}\left\{\left(\frac{V_{\mathrm{A}}}{V_{1}}\right)\left(1-v_{2}\right)+\frac{v_{2} V_{\mathrm{A}}}{x \bar{V}}\right. \\
& -\frac{1}{\zeta} \ln v_{2}+\frac{2 \sigma_{\mathrm{ec}}}{R T_{\mathrm{m}} \zeta}-\frac{1}{\zeta} \ln \left(\frac{x-\zeta+1}{x}\right) \\
& \left.\quad-\ln X_{\mathrm{A}}-\chi^{\prime}\left(1-v_{\mathrm{A}}\right)^{2}\right\}
\end{aligned}
$$

where $T_{\mathrm{m}}{ }^{\circ}$ is the equilibrium melting temperature of a pure polymer, $T_{\mathrm{m}}$ is the melting temperature of the system and $v_{\mathrm{A}}=v_{2} \chi_{\mathrm{A}}\left(V_{\mathrm{A}} / \bar{V}\right)$. The usual approximation of $\Delta f_{\mathrm{u}}\left(T_{\mathrm{m}}\right)=\Delta h_{\mathrm{u}}\left(T_{\mathrm{m}}{ }^{\circ}-T_{\mathrm{m}}\right) /$ $T_{\mathrm{m}}{ }^{\circ}$ has been made, where $\Delta h_{\mathrm{u}}$ is the enthalpy of fusion per repeating unit. When $x$ is large, and furthermore if $\zeta \ll x$ and $v_{1} \rightarrow 1$, eq 8 can be approximated

$$
\begin{aligned}
\frac{1}{T_{\mathrm{m}}} & -\frac{1}{T_{\mathrm{m}}{ }^{\circ}} \cong \frac{R}{\Delta h_{\mathrm{u}}}\left[\frac{2 \sigma_{\mathrm{ec}}}{R T_{\mathrm{m}} \zeta}+\left(\frac{V_{\mathrm{A}}}{V_{1}}\right)\left(1-\chi_{1}\right)\right. \\
& \left.-\ln X_{\mathrm{A}}-\frac{1}{\zeta} \ln v_{2}\right]
\end{aligned}
$$

where $\chi_{1}=B^{\prime} V_{1} / R T_{\mathrm{m}}$. If it may be allowed that $T_{\mathrm{m}} \mathrm{g}$ is equal to $T_{\mathrm{m}}$, eq 9 predicts that $1 / T_{\mathrm{m}} \mathrm{g}$ is proportional to $\ln v_{2}$. However, eq 9 does not predict the linear dependence of $1 / T_{\mathrm{m}}{ }^{\mathrm{g}} v \mathrm{~s} . \ln v_{2} v$ that has been found in this study. A similar situation also exists between $T_{\mathrm{m}} \mathrm{g}$ and the equilibrium melting temperature $T_{\mathrm{m}}{ }^{\mathrm{e}}$. Newman, Krigbaum, and Carpenter ${ }^{10}$ compared their melting temperature data on cellulose nitrate-ethanol gel with the theoretical prediction, eq 10 , for a homopolymer-diluent system.

$$
\frac{1}{T_{\mathrm{m}}{ }^{\mathrm{e}}}-\frac{1}{T_{\mathrm{m}}{ }^{\circ}}=\frac{R}{\Delta h_{\mathrm{u}}} \frac{V_{\mathrm{u}}}{V_{1}}\left(v_{1}-\chi_{1} v_{1}^{2}\right)
$$

They found that the numerical agreement between eq 10 and the experiments was not quite satisfactory. When their data are treated by plotting $1 / T_{\mathrm{m}}^{\mathrm{g}}$ against $\ln v_{2}$, good straight lines are obtained, too. ${ }^{13}$ Moreover, for the samples IIA-3 and IIC-3, which are the same in $X_{\mathrm{A}}$ but differ in $x$, the linear relationship between $1 / T_{\mathrm{m}} \mathrm{g}$ vs. $\ln v_{2} x$ holds. ${ }^{13}$ This is the reason why eq 10 did not fit the experimental results, since not only $\ln v_{2}$ but also $\ln v_{2} x$ terms are not contained in the equation. However, the discrepancy between theory and experiments does not mean that the theory is inadequate, rather it indicates the necessity to consider the meaning of $T_{\mathrm{m}} \mathrm{g}$ more precisely. Doty, Wagner and Signer ${ }^{14}$ reported the light-scattering study of the PVCdioxane system, and have shown that the presence of stable aggregates in the solution. The same conclusion has also been reached for gelatin - water ${ }^{9}$ and cellulose nitrate-ethanol gels. ${ }^{10}$

Newman, et al., also discussed the reasons for the disagreement, and pointed out that the crystallites in the gel are in equilibrium with aggregates, rather than single molecules as assumed by theory. This is the most important point for the gelarion and the gel melting. Thus, the melting temperature of gel is just the point at which sol-gel transformation occurs, and the sol still contains many crystallites. Therefore, 


\section{A. Takahashi, T. Nakamura, and I. Kagawa}

the melting temperature of gel does not correspond to the thermodynamic singurality where the last trance of crystallite has melted away. This was also pointed out by Ziabicki. ${ }^{15}$ Thus, the discriminative identification that $T_{\mathrm{m}}=T_{\mathrm{m}} \mathrm{g}$ is not accepted.

To form the three dimensional network structure, at least a polymer molecule must pass through two different crystallites, and the situation is quite similar to that of gel formation by chemical cross-linking reaction, though no chemical reactions actually happen. The free energy of formation of a crystallite from a copolymer diluent system is given by ${ }^{16}$

$$
\Delta F\left(v_{2}, X_{\mathrm{A}}\right)=2 \zeta \sigma_{\mathrm{u}}(\pi \rho)^{1 / 2}-\Delta F_{\mathrm{f}}\left(\zeta, \rho, X_{\mathrm{A}}\right)
$$

Here, it is supposed that the crystallite comprise $\zeta$ units in length and $\rho$ crystalline sequences in cross section, and $\sigma_{\mathrm{u}}$ in the equation is the lateral surface free energy. From eq 7 , we find, when $x$ is large and $\zeta \ll x$, that

$$
\begin{aligned}
\frac{\Delta F_{\mathrm{f}}}{x N}= & \left(\frac{\zeta \rho}{x N}\right)\left\{\Delta f_{\mathrm{u}}+R T\left[\frac{V_{\mathrm{A}}}{V_{1}}\left(-v_{1}\right)\right.\right. \\
& +\frac{1}{\zeta} \ln v_{2}-\frac{2 \sigma_{\mathrm{ec}}}{R T \zeta}+\ln X_{\mathrm{A}} \\
& \left.\left.+\left(\frac{V_{\mathrm{A}}}{V_{1}}\right) \chi_{1} \frac{\left[1-v_{2} X_{\mathrm{A}}\left(V_{\mathrm{A}} / \bar{V}\right)^{2}\right]}{\left[1-v_{2}\left(V_{\mathrm{A}} / \bar{V}\right)(\zeta \rho / x N)\right]}\right]\right\}
\end{aligned}
$$

Since $\zeta \rho / x N \ll 1$, and furthermore when the system is dilute, so that $v_{2} \rightarrow 0$, eq 12 can be expressed as

$$
\begin{aligned}
\Delta F_{\mathrm{f}}= & \zeta \rho\left[\Delta f_{\mathrm{u}}-\frac{2 \sigma_{\mathrm{ec}}}{\zeta}-R T\left(\frac{V_{\mathrm{A}}}{V_{1}}\right)\left(1-\chi_{1}\right)\right. \\
& \left.+R T \ln X_{\mathrm{A}}+\frac{R T}{\zeta} \ln v_{2}\right]
\end{aligned}
$$

Assuming that $\sigma_{\mathrm{u}}$ is quite small so that the lateral surface free energy term may be neglected, eq 11 can be rewritten as

$$
\begin{aligned}
& \Delta F\left(v_{2}, X_{\mathrm{A}}\right) \equiv \rho \Delta F_{\zeta}=\rho\left[2 \sigma_{\mathrm{ec}}-\zeta \Delta f_{\mathrm{u}}\right. \\
& \left.\quad+\zeta R T\left(\frac{V_{\mathrm{A}}}{V_{1}}\right)\left(1-\chi_{1}\right)-\zeta R T \ln X_{\mathrm{A}}-R T \ln v_{2}\right]
\end{aligned}
$$

Therefore, $P_{\zeta}=\exp \left(-\Delta F_{\zeta} / R T\right)$ may be regarded as the probability that an $A$ unit, which is being added to a specified site on the lateral surface of the crystallite from the specified composition, is suitably situated within a sequence of length $\zeta .^{17}$ Thus, there will be $(x-\zeta+1) P_{\zeta} \cong$ $x P_{\zeta}$ crystallized sequences per average chain when $\zeta \ll x$. If $(x-\zeta+1) P_{\zeta} \ll 1$, the chance that a chain $x$ unit long contains a crystallized sequence is just $(x-\zeta+1) P_{\zeta}$. The expectancy $\Omega$ that the chain, which has passed through a crystallite, has crystalline sequences, so that it will enter to yer another crystallites, will be

$$
\Omega=(x-2 \zeta+1) P_{\zeta} \cong x P_{\zeta}
$$

Here, we have neglected the entropy problem associated with the distribution of the crystalline sequences into numerous crystallites. Equation 15 is just the form given for the condition of gel formation. ${ }^{4}$ If $\Omega>1$, the system will be lead to gelation and if $\Omega<1$, only the sol fraction will be formed in the system. Therefore, the critical condition is given by $\Omega=1$, and the gel melting temperature $T_{\mathrm{m}} \mathrm{g}$ is the temperature at which $\Omega=1$. Letting $\Omega=1$ in eq 15 , and taking logarithm, we obtain

$$
\begin{aligned}
& \frac{1}{T_{\mathrm{m}}^{\mathrm{g}}}-\frac{1}{T_{\mathrm{m}}{ }^{0}}=\frac{R}{\Delta h_{\mathrm{u}}}\left[\frac{2 \sigma_{\mathrm{ec}}}{R T_{\mathrm{m}} \mathrm{g} \zeta}\right. \\
& \left.\quad+\left(\frac{V_{\mathrm{A}}}{V_{1}}\right)\left(1-\chi_{1}\right)-\ln X_{\mathrm{A}}-\frac{1}{\zeta} \ln v_{2} x\right]
\end{aligned}
$$

Since $\chi_{1}=B^{\prime} V_{1} / R T_{\mathrm{m}}^{\mathrm{g}}$, eq 16 can be rewritten as

$$
\begin{aligned}
\frac{1}{T_{\mathrm{m}} \mathrm{g}}= & \frac{\zeta}{\left(\zeta \Delta h_{\mathrm{u}}+\zeta B^{\prime} V_{\mathrm{A}}-2 \sigma_{\mathrm{ec}}\right)} \\
& \cdot\left(\frac{\Delta h_{\mathrm{u}}}{T_{\mathrm{m}}{ }^{0}}+\frac{R V_{\mathrm{A}}}{V_{1}}-R \ln X_{\mathrm{A}}\right) \\
& -\frac{R}{\left(\zeta \Delta h_{\mathrm{u}}+\zeta B^{\prime} V_{\mathrm{A}}-2 \sigma_{\mathrm{ec}}\right)} \ln v_{2} \dot{x}
\end{aligned}
$$

Equation 17 is the form that is found by these experimental results, and the meaning of the intercept and the slope in the empirical equation 6 is now clear. Generally, $\zeta$ increases with the increase of the temperature, and $\sigma_{\mathrm{ec}}$ is also expected to vary with the temperature. However, judging from the plots shown in Figure 7 and eq 17 , both $\zeta$ and $\sigma_{\text {ec }}$ may be regarded as practically independent of the temperature and also of molecular weights of the samples. If $T_{\mathrm{m}}{ }^{\circ}$, $\Delta h_{\mathrm{u}}, B^{\prime}$, and $X_{\mathrm{A}}$ are known, we may estimate $\zeta$ and $\sigma_{\mathrm{ec}}$ from the plots of $1 / T_{\mathrm{m}} \mathrm{g} v s . \ln v_{2} x$. First, we evaluate the value of $\left(\zeta \Delta h_{\mathrm{u}}+\zeta B^{\prime} V_{\mathrm{A}}-\right.$ 
$\left.2 \sigma_{\mathrm{ec}}\right)$ from the slope of the plot. Then, inserting it, together with the values of $T_{\mathrm{m}}{ }^{\circ}, \Delta h_{\mathrm{u}}, B^{\prime}$, and $X_{\mathrm{A}}$, into the expression of intercept, we may determine $\zeta$. Finally, $\sigma_{\mathrm{ec}}$ may be estimated from the values of $\left(\zeta \Delta h_{\mathrm{u}}+\zeta B^{\prime} V_{\mathrm{A}}-2 \sigma_{\mathrm{ec}}\right)$ and $\zeta$.

Nakajima and Hamada ${ }^{18}$ measured the melting point depression of the $\mathrm{PVC}$-diluent systems, and reported that $T_{\mathrm{m}}=583^{\circ} \mathrm{K}$ for the sample of $\alpha=0.77$ and $T_{\mathrm{m}}=558^{\circ} \mathrm{K}$ for the sample of $\alpha=0.64$. Since $T_{\mathrm{m}}{ }^{\circ}$ in eq 17 is defined as the equilibrium melting temperature of the pure polymer, we estimated it from the extrapolation of those $T_{\mathrm{m}}$ values to $\alpha=1$ according to the equation of the melting point depression of a copolymer. ${ }^{12}$ The result thus obtained was $T_{\mathrm{m}}{ }^{\circ}=628^{\circ} \mathrm{K}$. For the enthalpy of fusion, they obtained the value of $785 \mathrm{cal} / \mathrm{mol}$ irrespective of the syndiotacticity. The values of $\boldsymbol{B}^{\prime}$ for $\mathrm{PVC}$-anisole, dioxane, and EDC were calculated from the data given by Doty and Zable. ${ }^{8} \quad X_{\mathrm{A}}$ is identified with $\alpha$, and their average value was calculated from Table I. The results of the analysis are shown in Table II. The values of $\zeta$ thus estimated are 10 to 12 .

Harrison, Morgan and Park $^{3}$ have pointed out that the small runs of stereoregular (syndiotactic) sequences in the PVC chain are responsible for the formation of the crystallite region and estimated that, on the average, a random atactic polymer of molecular weight 30000 would contain about 1 regular sequence of 11 syndiotactic units in each polymer chain.

From the NMR measurements on poly $\left(\beta-d_{2}\right.$-vinyl chloride), Enomoto, Asahina and Sato ${ }^{19}$ estimated that the number-average syndiotactic sequence length is about 20 , and also showed that it does not vary appreciably with the polymerization temperature. These sequeuce lengths may be taken as a support for the validity of the present analysis to estimate the value of $\zeta$ from $T_{\mathrm{m}}{ }^{\mathrm{g}}$.

In Table II, the values of $\sigma_{\mathrm{ec}}$ are also shown. For the system of PVC-EDC, the small positive value of $\sigma_{\mathrm{ec}}$ was obtained, whereas the small but negative $\sigma_{\mathrm{ec}}$ were evaluated for the other two systems. According to the definition of $\sigma_{\mathrm{ec}}$, it must be a positive value. One of the reasons for the negative $\sigma_{\mathrm{ec}}$ may reside in the selection of $T_{\mathrm{m}}{ }^{\circ}$. Since $T_{\mathrm{m}}{ }^{\circ}=628^{\circ} \mathrm{K}$ has not been evaluated by extrapolation to $x \rightarrow \infty$ and $\zeta \rightarrow \infty$, it is expected that the true equilibrium melting temperature will be much higher than $628^{\circ} \mathrm{K}$. To see the effect of the selection of $T_{\mathrm{m}}{ }^{\circ}$ on the sign and magnitude of $\sigma_{\mathrm{ec}}$ as well as on the value of $\zeta$, let us assume $700^{\circ} \mathrm{K}$ as $T_{\mathrm{m}}{ }^{\circ}$. Then, $\sigma_{\text {ec }}=$ $-222,-4$, and $+236 \mathrm{cal} / \mathrm{mol}$ are results of the PVC-anisole, dioxane and EDC systems, respectively, whereas $\zeta=12.2,9.7$, and 9.9 are obtained for the same systems. Even though such a high value of $T_{\mathrm{m}}{ }^{\circ}$ was employed, the sign of $\sigma_{\mathrm{ec}}$ did not change. However, it appears that the value of $\zeta$ is not seriously affected by the selection of $T_{\mathrm{m}}{ }^{\circ}$. From the comparison between the values of $\left(\zeta \Delta h_{\mathrm{u}}+\zeta B^{\prime} V_{\mathrm{A}}\right)$ and $\sigma_{\mathrm{ec}}$ shown in Table II, it is apparent that, when $\zeta \geq 10$, the absolute value of $\sigma_{\mathrm{ec}}$ is at most only $4 \%$ of the value of $\left(\zeta \Delta h_{\mathrm{u}}+\zeta B^{\prime} V_{\mathrm{A}}\right)$. Considering the fact that the appreciable scattering of the experimental points is observed for the plots shown in Figure 7 , the determination of the values of both the intercept and the slope of the plots within an accuracy of several $\%$ is difficult. Moreover, since the true $T_{\mathrm{m}}{ }^{\circ}$ is not available at present, we must conclude that the precise determination of $\sigma_{\mathrm{ec}}$, including its sign, is quite impractical for the present case.

It is apparent that eq 17 fairly well represents the experimentally observed gel melting points as functions of molecular weight, concentration of copolymer, and the thermodynamic nature of solvents.

Acknowledgment. We are indebted to Dr. Shuji Ozawa, Teijin Co. for his kind supply of the PVC samples, and also to Mr. Hokaku, Japan Spectroscoptic Co. Ltd·, for his assistance in performing the IR measurements.

\section{REFERENCES}

1. A. Takahashi, M. Obara, and I. Kagawa, Kogyokagaku Zashi (J. Chem. Soc. Japan Ind. Chem. Sect.), 66, 960 (1963).

2. A. T. Walter, J. Polym. Sci., 13, 207 (1954).

3. M. A. Harrison, P. H. Morgan, and G. S. Park. IUPAC Symposium on Macromolecular Chemistry, Leiden, 1970.

4. D. R. Paul, J. Appl. Polym. Sci., 11, 439 (1967).

5. P. J. Flory, "Principles of Polymer Chemistry," Cornell University Press, Ithaca, N. Y. 1953, chapter IX.

6. J. E. Eldridge and J. D. Ferry, J. Phys. Chem., 
58, 992 (1954).

7. H. Germer, K. H. Hellwege, and U. Johnson, Makromal. Chem., 60, 106 (1963).

8. P. Doty and H. S. Zable, J. Pnlym. Sci., 1, 90 (1946); P. Doty and E. Mishuck, J. Amer. Chem. Soc., 69, 1631 (1947).

9. H. Boedtker and P. Doty, J. Phys. Chem., 58, 968 (1954).

10. S. Newman, W. R. Krigbaum, and K. K. Carpenter, J. Phys. Chem., 60, 648 (1956).

11. L. Mandelkern, "Crystallization of Polymers," McGraw-Hill, New York, N.Y. 1964, p 112.
12. P. J. Flory, J. Chem. Phys., 17, 223 (1949).

13. to be published.

14. P. Doty, H. Wagner, and S. Singer, J. Phys. Chem., 51, 32 (1947).

15. A. Ziabicki, Sen-i Gakkaishi, 26, 156 (1970).

16. L. Mondelkern, J. Appl. Phys., 26, 443 (1955).

17. P. J. Flory, Trans. Faraday Soc., 51, 848 (1955).

18. A. Nakajima, H. Hamada, and S. Hayashi, Makromol. Chem., 95, 40 (1966).

19. S. Enomoto, M. Asahina, and S. Sato, J. Polym. Sci., Part A-1, 4, 1373 (1966). 